\title{
Investigation of the casualties of terrorism events in Columbia
}

\author{
Junfang Zhu' ${ }^{1,}$, Peijie $\mathrm{Ma}^{1}$, Xlaoyan Sun ${ }^{2}$, , Benxian $\mathrm{Li}^{3,4}$ \\ ${ }^{1}$ School of Science, Southwest University of Science and Technology, Mianyang 621010, China \\ ${ }^{2}$ College of Physics and Electronic Engineering, Guangxi Teacher Education University, Nanning \\ 530023, China \\ ${ }^{3}$ School of Information System and Management, National University of Defense Technology, \\ Changsha, 410073, China \\ ${ }^{4}$ Officers College of Chinese Army Police Force, Sichuan Chengdu, 610213, China

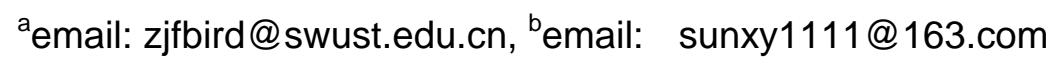

Keywords: Terrorism events; statistic feature; casualty

\begin{abstract}
In this report, terrorism events are investigated. We make statistic analysis for the distribution of fatality number and injury number by the data from open database GTD. The power law distribution is found which exists not only for the whole Colombia country but also the large organizations in this country. For Colombia, the power law distribution of fatality number and injury number is mainly composed of power law distribution of organizations in this country. Besides, it is found that, the sine function relationship exists between average activity degree and average severity (casualty number) of organizations except two organizations with big severity and two organizations with low average activity degree. The results will shell light on the anti-terror work.
\end{abstract}

\section{Introduction}

In recent decades, the burst of global terrorism events is focused on comprehensively [1]. There has been a lot of research developed in order to analyze the causes $[2,3,4,5]$ and understand the mechanism of terrorism $[6,7,8]$. Generally, the terrorist events are regarded as disordered, chaotic and unpredictable. Although the paper [9] gives early warning signal, the casualties are still unexpected. But, in fact, some common characteristics of casualties exist for different terrorist events induced by regardless of what reasons. These common characteristic is investigated from three different lays. First of all, the research is done from the view of global wide. For example, the research of Richardson and Cederman has shown the frequency as a function of severity follows a power-law distribution [10]. Further Aaron Clauset demonstrates the robust property of the scale invariance for different weapon types in global terrorist events [11]. Secondly, terrorism is studied from the scope of country. The robust property of scale invariance is found for different countries through the data statistics [12, 13, 14,]. Finally, some investigation is from the lay of organization. Aaron Clauset the distributions of event severities are shown to collapse onto a heavy-tailed distribution [15]. Whether does the same distribution of power law hold in different lays of a country? Moreover, the activity degree of organizations is never considered in the research of terrorism. Whether does the casualty number of an organization be influenced by the activity degree?

This paper is organized as follows. In Section 2, the statistic feature of the casualty number is studied for Colombia. In Section 3, we analyze the statistic feature of casualty number for several large organizations to detect the main origin of the statistic feature of casualty number for Colombia. In section 4, we discuss the relationship of average activity degree and average casualty number and provide proposal to anti-terror. Finally, the conclusion is given in section 5. 


\section{The statistic feature of the casualties number for Colombia}

First of all, we investigate the frequency distribution as a function of casualties for the Colombia and several large groups in Colombia. Our data is from the database the global terrorism database (GTD) [7].This open-source database contains terrorist events around the world from 1970 through 2013 (with annual updates planned for the future). There is a simple description for every event providing the data, country, city, perpetrator, fatality, injured and target type.

For Colombia, the number of fatalities and injured versus temporal series is shown in Fig. 1 . The largest number of fatalities and the injured is indicated by an arrow. Obviously, the number of fatalities and the injured is different for every event. We can see there are a relatively low percentage for the event with large number of fatalities and the injured. By calculation, we obtain the high standard deviations $\sigma=8.15$ and $\sigma=6.22$ for the number of fatalities and injured. These results indicate that the distribution is not uniform.

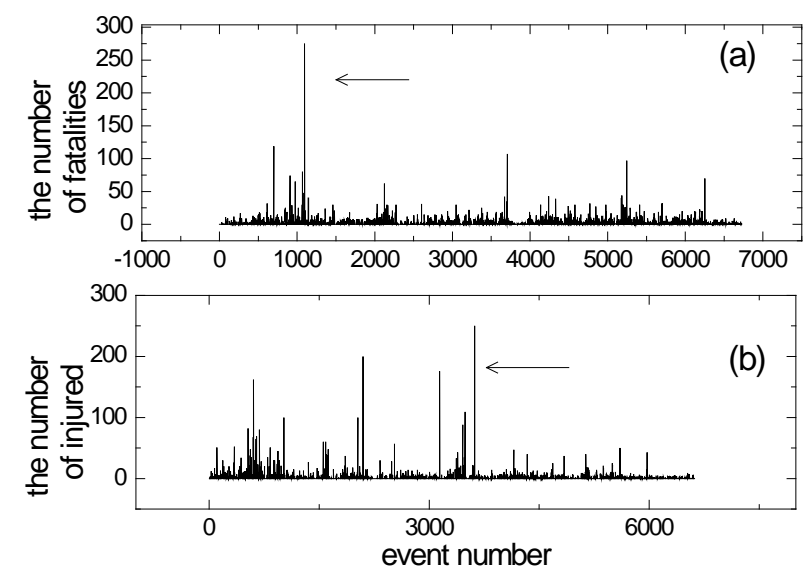

Fig. 1. the number of fatality and injured for Colombia

In order to make the statistic character clear, we exploit Zipf's plot [8] to analyze the statistic distribution feature for the 7181 events in which 6727 events induce the fatality or the injured. Zipf's plots of the number of fatality and injured are shown in Fig. 2. Notably, it follows the power law distributions. To find stationary rank exponent $\alpha_{z}$, the fitting is carried out by the least-square fit method after a cutoff in the tail. After we remove some points in the tail, the rank exponent $\alpha_{z}=0.573$ and $\alpha_{z}=0.752$ are obtained for the fatality and injured respectively as shown in Fig. 2. In fact, Zipf's plot can be considered a cumulative distribution with power law feature and the relationship between the exponent Zipf's rank exponent $\alpha_{z}$ and the power law exponent $\alpha$ is as following [9]

$$
\alpha=1+\frac{1}{\alpha_{z}}
$$

So we have $\alpha=2.73$ and $\alpha=2.33$ which are just corresponding with the feature of non_G7 countries [10]. But these events are executed by many organizations in Colombia. Then, we want to know if the power law property can extend to the events executed by the organizations 

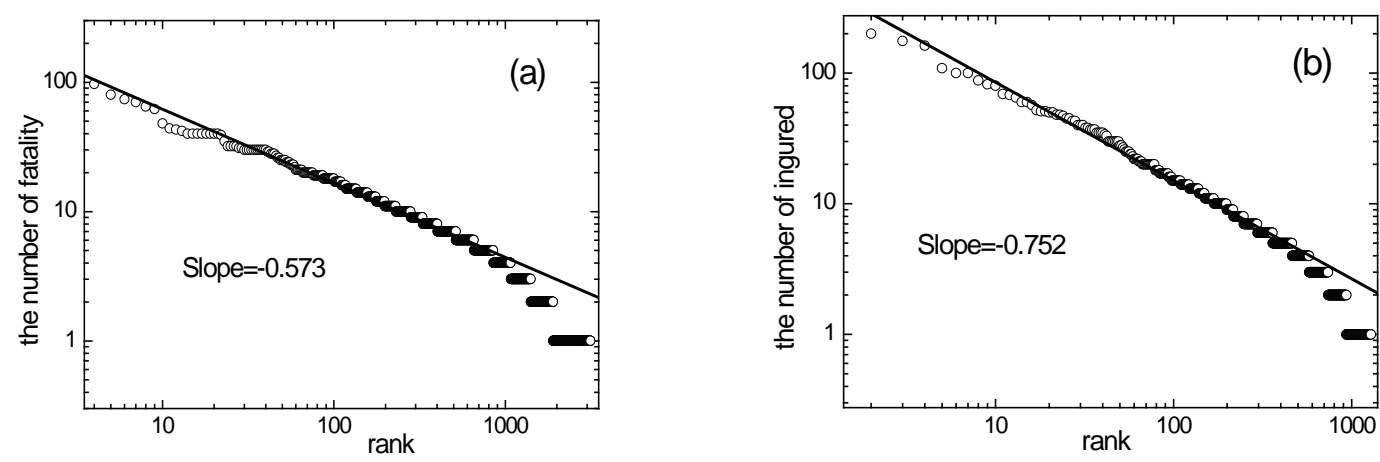

Fig. 2. The Zipf's plot of the terrorism events for Colombia

\section{The statistic feature of casualties number for several large organizations and the main origin of the statistic feature of casualties number for Colombia}

In the 7181 terrorism events in Colombia, 1818 events is perpetrated by Revolutionary Armed Forces of Colombia (FARC) and 1220 events by National Liberation Army (ELN). For Colombia and every large organization, the number of fatalities and the injured is shown in the table 1.

Table 1 the number of fatalities and injured induced by Colombia and large organizations.

\begin{tabular}{|l|l|l|l|l|l|l|l|}
\hline & Colombia & FARC & ELN & $\begin{array}{l}\text { death } \\
\text { squad }\end{array}$ & M19 & $\begin{array}{l}\text { popular } \\
\text { liberation army }\end{array}$ & $\begin{array}{l}\text { Narco } \\
\text { terrorist }\end{array}$ \\
\hline fatalities & 13271 & 4992 & 1761 & 350 & 1303 & 506 & 427 \\
\hline injured & 8356 & 3202 & 1042 & 82 & 495 & 144 & 797 \\
\hline
\end{tabular}

In Colombia, FARC and ELN are the two large organizations. Revolutionary Armed Forces of Colombia (FARC) is the largest, best trained, and best equipped guerrilla organization in Colombia. There are approximately 7000 armed combatants and an unknown number of supporters, mostly in rural areas. The group members execute armed attacks against Colombian political and military targets.

National Liberation Army (ELN) is a Rural-based, anti-US, Maoist-Marxist-Leninist guerrilla group formed in 1963. Attempted peace talks with the government ended in May 1992. At least there are 3000 combatants.

From table 1, we find that the most fatalities and injured are perpetrated by these large organizations specifically FARC and ELN. Next, we mainly investigate statistic feature of the events carried on by the organizations FARC and ELN.

For the events perpetrated by FARC and ELN, the power law distribution of the number of fatalities still can be observed as shown in Fig. 3(a). We have obtained the number distribution of fatalities with Zipf's exponents $\alpha_{z}=0.616$ and $\alpha_{z}=0.575$ for FARC and ELN with different cutoffs respectively. That is to say there are power law exponents $\alpha=2.623$ and $\alpha=2.739$. Besides, we also explore the other organizations with more than 100 events (including death squad, M19, Narco terrorist, Popular Liberation Army and so on). It is found that the power law form is preserved for the distribution of the number of fatalities and the power law exponents is between $\alpha=2.43$ and $\alpha=2.75$. Obviously, the results demonstrate the distribution of the number of fatalities can be described by power law form not only for the whole Colombia country but also different organizations in this country. Due to the two-thirds of the number of fatality is evoked by the organizations in table 1 , we think simply the power law of main big organizations is origin of power law of Columbia for fatalities.

Similarly, we study the distribution of the number of injured for FARC and ELN. From Zipf's rank plot we see that the power law distribution still holds as shown in Fig.3 (b). By the least-square fit method we obtain the Zipf's exponents $\alpha_{z}=0.787$ and $\alpha_{z}=0.760$ corresponding with the 
power law exponents $\alpha=2.27$ and $\alpha=2.32$ with a tail cutoff. It is surprising that the same statistic feature appears for the other organizations only with different power law exponents. So there is the same results with the distribution of the number of fatality for the number of injured.
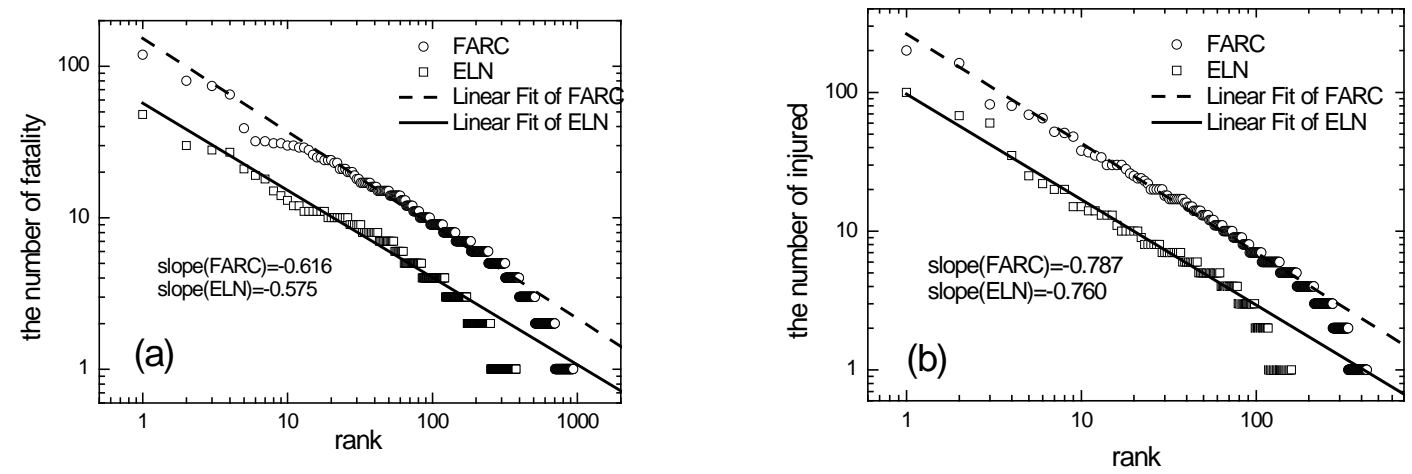

Fig. 3. The Zipf's plot of the terrorism events for FARC and ELN

The relationship of average activity degree and average casualty number and the proposal to anti-terror.

Although the results above tell us the statistic feature, the severity (casualties) itself is more focused in anti-terror. So we next explore the relationship of average severity and average activity degree [11] for every organization in Colombia.

The average activity degree is defined as the average time interval of execute terrorism event for every terrorism organization. From Fig. 4(a), We found two big severity with about 13.4 and 38.6 responding to the organization Liberation Tigers of Tamil Eelam (LTTE) and Islamic State of Iraq (ISI) and two big average activity degree with about 95.3 and 98.7 responding to the organization Mujahideen-I-Khalq (MK) and Popular Front for the Liberation of Palestine (PFLP) which is signed by hollow circle. For the former two organizations, LTTE has very big strength of about 13000-16000 combatants and ISI is the participator of Iraq war belonging to global terrorism events. These two organizations should be the key. For the later two organizations, we still don't neglect them even though they executed a terrorism event for long time. After cut out these four points signed by hollow circle, we fit the curve into sin function shown in Fig. 4(b). This indicates that for ordinary organization in Colombia, the organizations with medium average activity degree can caused more big severity than the ones with low and higher average activity degree. So, for these ordinary organizations, the key of anti-terror should be on the organization with medium activity degree.
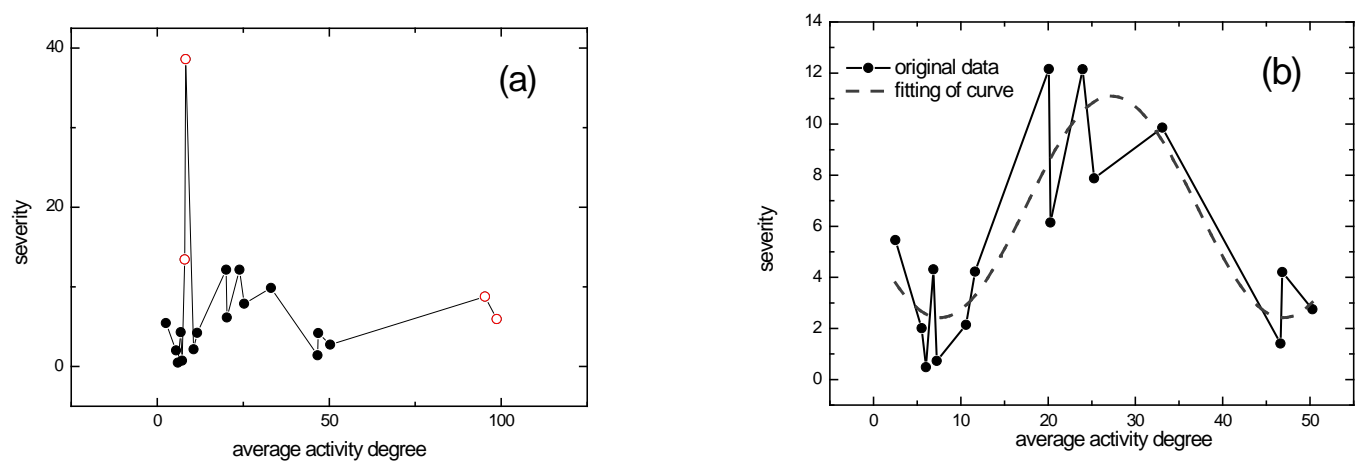

Fig.4. The average severity vs. average activity degree for Colombia. 


\section{Conclusion}

Although the results above tell us the statistic feature, the severity (casualties) itself is more focused in anti-terror. So, in the following study, we will explore the relationship of average severity and average activity degree [11]\} for every organization in Colombia.

The average activity degree is defined as the average time interval of execute terrorism event for every terrorism organization. From Fig. 4(a), we found two big average severity with about 13.4 and 38.6 corresponding to the organization Liberation Tigers of Tamil Eelam (LTTE) and Islamic State of Iraq (ISI) and two big average activity degree with about 95.3 and 98.7 corresponding to the organization Mujahideen-I-Khalq (MK) and Popular Front for the Liberation of Palestine (PFLP) which is signed by hollow circle. For the former two organizations, LTTE has very big strength of about 13000-16000 combatants and ISI is the participator of Iraq war belonging to global terrorism events. These two organizations should be the key. For the later two organizations, we still don't neglect them even though they executed a terrorism event for long time. After cut out these four points signed by hollow circle, we fit the curve into sin function shown in Fig. 4(b). This indicates that for ordinary organization in Colombia, the organizations with medium average activity degree can caused more big average severity than the ones with low and higher average activity degree. So, for these ordinary organizations, the key of anti-terror should be on the organization with medium average activity degree.

\section{Acknowledgement}

In this paper, the research was sponsored by the Nature Science Foundation of Henan Province (Project No. 201112400450401) and Youth Fund Project of Luoyang Institute of Science and Technology (Project No. 2010QZ16) , the School Fund under grant No. 10zx7138.

\section{References}

[1] Eliot M, Parsing terrorism, Science, 2012, 336, 820-823.

[2] Krisztina K, Helge L, Günther G. Schulze, on the origin of domestic and international terrorism, European journal of Political Economy, 2011,17, S17-s36

[3] Ghulam A, Khalid Z, Tan S, Faiza S, Exploring the root causes of terrorism in South Asia: everybody should be concerned, Qual Quant, 2014,48, 3065-3079

[4] Alan B. Krueger and Jitka Maleková, Attitudes and Action: Public Opinion and the Occurrence of International Terrorism, Science, 2009, 325, 1534.

[5] Bradley T. Kerridge, Maria R. Khan, Júrgen Rehm, Amir Sapkot, Terrorism, civil war and related violence and substance use disorder morbidity and mortality: A global analysis, Journal of Epidemiology and Global Health, 2014, 4, 61-72.

[6] Johnson N F, Spagat M, Restrepo J A, et al. Universal patterns underlying ongoing wars and terrorism [EB/OL]. (2006-03-03) [2012-01-10]. http://xxx.lanl.gov/abs/physics/0605035.

[7] Galam S. The September 11 attack: a percolation of individual passive support [J], Eur Phys J B, 2002, 26(1), 269-272.

[8] Clauset A, Heger L, young $\mathrm{M}$, et al. The strategic calculus of terrorism: substitution and compedition in the Isrial-Palastine Conflict [J], Cooperation and Conflict, 2010, 45(1), 6-33.

[9] Andy Martens, Raazesh Sainudiin, Chris G. Sibley, Jeff Schimel, David Webber, Terrorist Attacks Escalate in Frequency and Fatalities Preceding Highly Lethal Attack, Plos One, 2014, 9(4), e93732. 
[10] Richardson L F, Amer.Stat.Assoc. 1948, 43, 523-546

[11] Clauset A, Young M. Scale in variance in global terrorism [ES/OL]. (2005-05-010 [2012-01-10]. http://xxx.lanl.gov/abs/physics/0502014

[12] Clauset A, Young M and Gleditsch K S, J.Conlict Resolution 2007, 51(1), 58

[13] Güngör Gündöz, Dynamics of the world terro and the war in Iraq, Physica A 2007,376, 579-595.

[14] Jose Alvarez-Ramirez, Eduardo Rodriguez, Rafael Urrea, "Scale invariance in the 2003-2005 Iraq conflict”, Physica A 2007, 377, 291-301.

[15] Aaron Clauset, Kristian Skrede Gleditsch, THe developmental dynamics of terrorist organizations, arXiv: 0906.3287V3 [physics.soc-ph] 22 Nov 2012

[16] http://www.start.umd.edu/gtd/

[17] Zipf G K 1949 Human Behavior and the Principle of Least Effort: An Introduction to Human Ecology (Cambridge, MA: Addison-Wesley)

[18] Zhu J F, Han X p and Wang B H, "Statistical Property and Model for the Inter-Event Time of Terrorism Attacks”, Chinese Physics Letter, 2010, 27(6), 068902.

[19] Clauset A, Young M, physics/0502014, 2005 